\title{
Limitations of Neurobiological Approach in Neuropsychiatry: DCD and the Two Visual Systems Theory
}

Young-lim Lee*

Psychology department, Dankook University, Korea

\begin{abstract}
The purpose of this review is to address possible limitations of the neurobiological approach to understanding psychiatric disorders. Neurobiological approach helps to resolve the mind-body dualism and develop new assessment and treatment approaches in psychiatry. However, it could be a problem to place too much emphasis on certain aspects of neurobiology, specifically structural neuroanatomy, because of the complexity or comorbidity of neuropsychiatric disorders. Developmental Coordination Disorder (DCD), for instance, is generally related to problems in motor skills and this movement disability is often related to perception. One account, two visual systems theory, relied on functional distinction in brain; ventral stream is responsible for visual recognition (perceptual representation), and dorsal stream is responsible for the guidance of actions. Numerous neuropsychological and neurophysiological studies have suggested that there are two separate visual streams. What then can we understand of DCD from this neuroanatomical approach? Studies are now showing that shape perception is relevant to visually guided action, such as reaching-to-grasp an object. In retrospect, it is not so surprising that we would need information about 3D shape to interact with 3D structure. In this article, I reviewed fundamental findings of two-visual system theory and suggested problems of visually guided action to consider what shape perception implies for the hypothesis that there are two separate visual streams in the brain. Questions raised highlight possible limitations of adopting a structural neuroanatomical approach to account for perception and action effects, and by extent related psychiatric conditions such as DCD. In conclusion, neurobiological approach in neuropsychiatry, while useful, would be limited if it focuses too much on anatomical distinction.
\end{abstract}

Keywords: Neuroanatomy; Neurobiology; Neuropsychiatry; DCD; Ventral stream; Dorsal stream; Perception/action

\section{Introduction}

Psychiatry has been continuously developing and adopted a neurobiological approach. Although both psychiatry and neurology rest on a foundation of clinical neuroscience, these two areas are subsequently separated and are typically practiced differently. Whereas neurology has traditionally focused on anatomical distinction of brain, psychiatry has focused on mental problems which rested on a foundation of mind-body dualism. Nevertheless, neuropsychiatry, a branch of treatment that deals with mental disorders attributable to diseases of the nervous system, has grown to understand a causality of brain to mind. Thus, neuropsychiatry has become a special subdivision of psychiatry and it is also related to a subdivision of neurology, which is neuropsychology and behavioral neurology that work on clinical problems of cognition and/or behavior caused by brain injury or brain disease. Among debates relating to neuropsychiatry, most of work agrees that mind and brain are not separable and neuropsychiatry could be more effective approach collaborating with neuropsychology, cognitive neuroscience, and neurosurgery in the field of psychiatry [1-4]. There is no doubt that these neurobiological approaches in psychiatry could help to develop new possible assessment and treatment using many tools (e.g., brain imaging, genetics, neurophysiology, neuropsychology, and neuro-psychopharmacology), but we should be cautious in focusing too much on discrete anatomical brain areas because it is hard to find unique brain areas associated with individual disorders.

Developmental Coordination Disorder (DCD) is typically related to problems with almost any sensory or motor skills, such as abnormalities in postural control $[5,6]$ as well as in fine motor skills [7]. But the disability has also been related to perception, such as a deficit in the mapping of visual and proprioceptive information $[8,9]$, difficulties in visuomotor integration [10] and abnormalities in the execution of movements without perceptual component [11]. These findings seem to suggest that DCD is a fairly generalized problem, affecting movement, as well as perception. Vision plays a critical role in our behavior, as well as the recognition of objects by providing us with information about the world. Numerous neurological studies including neurophysiological and neuropsychological studies have explored the neural mechanisms by which vision is functionally analyzed. One of the most intriguing theories of the visual system claims that there are two functionally distinct visual pathways. Milner and Goodale [12] proposed a functional difference between two visual systems: a ventral system mainly which mainly plays a role in object recognition (perceptual representation or identification) and a dorsal system which mainly plays a role in the visual guidance of actions. They distinguished between vision for perception and vision for action based on the separate functions of ventral and dorsal stream processes.

This article consists of two parts. First is to review the two visual systems theory based on neurophysiological and neuropsychological studies. Second is to examine possible problems for visually guided action in this two-system framework which highlight the limitations of neurobiological approach in neuropsychiatry.

*Corresponding author: Young-lim Lee, Psychology department, Dankook University, Korea, Tel: 82-107455554; E-mail: ppangi77@gmail.com

Received March 03, 2015; Accepted April 21, 2015; Published April 30, 2015

Citation: Lee Y (2015) Limitations of Neurobiological Approach in Neuropsychiatry: DCD and the Two Visual Systems Theory. J Psychiatry 18: 289 doi: 10.4172/2378-5756.1000289

Copyright: (c) 2015 Lee Y. This is an open-access article distributed under the terms of the Creative Commons Attribution License, which permits unrestricted use, distribution, and reproduction in any medium, provided the original author and source are credited 


\section{Two Visual Systems: The Ventral and the Dorsal Streams}

In the early eighties, Ungerleider and Mishkin [13] proposed, on the basis of studies in the macaque monkey, that anatomical separation of the cortical visual projections into two distinct streams reflects different functions in visual processing. A ventral stream leaves V1 and projects to the inferotemporal cortex, while a dorsal stream projects from V1 to the posterior parietal cortex. According to Ungerleider and Mishkin, the ventral stream is responsible for the identification and recognition of objects, while the dorsal stream is responsible for the localization of those same objects. Goodale and Milner [12,13] suggested a somewhat different interpretation of the functions of the dorsal stream, although their interpretation of the functions of the ventral stream is similar to that of Ungerleider and Miskin [14]. They proposed that the dorsal stream is mainly involved in the visual control and guidance of motor behavior rather than just spatial localization. They suggested that the major difference between the two streams is how each stream transforms the available visual information. In other words, the ventral stream transforms the visual information into an object-centered framework allowing recognition of the object, whereas the dorsal stream transforms the visual information into a viewer-centered framework allowing the viewer to control visuomotor behavior [15].

\section{Neuropsychological Evidence: Optic Ataxia vs. Visual Form Agnosia}

The initial evidence for the two visual systems theory has come from studies on patients who had severe brain damage in one stream or the other. On the one hand, there are patients where the posterior parietal cortex, a major part of the dorsal stream, is damaged. These patients suffer from optic ataxia, manifesting difficulties in making correct motor movements towards visually displayed targets. Observations on patients with optic ataxia due to damage to the dorsal system have shown that they have difficulties using visual information to control and guide motor behavior, while they have no difficulty in recognizing objects. Patients are able to neither reach toward an object in the correct direction nor adjust the orientation of their hand when reaching although they are able to verbally describe the orientation of the object [16]. In addition, such patients can also have difficulties in grasping objects with appropriate grip size and finger placements at appropriate points on the object's surface [17-19].

On the other hand, there are patients where the inferotemporal cortex, a major part of the ventral stream, is damaged. These patients suffer from visual form agnosia, manifesting difficulties in recognizing or describing objects, faces, drawings, or abstract designs, even though they have no difficulty in using visual information to control and guide motor behavior. Goodale et al. [20] studied orientation and size perception in patient DF. To investigate the dissociation between perceptual judgment of orientation and visuomotor control, they used a large slot presented at varying angles. When DF was asked to insert a card into the slot, she was able to orient her hand to match the orientation of the slot and insert the card accurately. However, when asked to report the orientation of the slot verbally or rotate the card to match the orientation of the slot without inserting it, she was unable to do so. In short, she can rotate her hand to match the orientation of the slot only when she attempts to insert the card into the slot. Her visuomotor control is intact but her perceptual judgment of orientation is impaired because the ventral system is damaged. Similar dissociation between perception and visuomotor behavior was found in a study of size perception. When she was asked to pick up blocks of different sizes, she had no trouble in picking up the blocks by adjusting the aperture between her index finger and thumb. Despite her ability to control her motor behavior, she was unable to report verbally if two small blocks were of the same or different widths. Additionally, when she was asked to use her index finger and thumb to make a perceptual judgment of the object's width, she was not able to make correct judgments. Those observations on both orientation and size perception tasks showed that DF has an intact dorsal system, but her ventral system is dysfunctional [12].

\section{Neurophysiological Evidence: Dorsal Stream vs. Ventral Stream}

The dorsal stream is located in the Posterior Parietal Cortex (PPC), including areas such as middle temporal (MT/V5), medial superior temporal (MST), Lateral Intraparietal Area (LIP), Anterior Intraparietal (AIP), among others [15]. Cohen and Andersen [20,21] reviewed the role of the PPC in movement planning. Within the PPC there are three areas: LIP, Parietal Reach Region (PRR) including the Medial Intraparietal Area (MIP), and AIP. These areas have been identified as being specified for different types of movement plans. LIP and PRR are specific for saccadic eye movements and reaching, respectively, [22] and AIP is specific for grasp planning [23]. Neuroimaging studies (fMRI) on humans have shown a specific activation in a region of PPC. Connolly et al. [24] found that neurons in a region located along the medial surface of the PPC responded preferentially during delayedreach trials in which the subject planned to point to a specific location as opposed to delayed-saccade trials in which the subject planned to make a saccade to that same location. Culham et al. [25] found that AIP is activated during grasping objects which requires to preshape the hand as compared to reaching which does not require preshaping or $2 \mathrm{D}$ images of objects for which grasping is not required.

An area in the caudal part of the intraparietal sulcus (area cIPS) also has been shown to have a role in the dorsal stream in object-directed action [26]. It is easy to recognize objects from different views. However, visual control of actions like grasping is likely to be quite viewpointdependent because the same object presented from different viewpoints often requires different hand postures during grasping. James et al [26] investigated the effect of viewpoints on visuomotor actions in area cIPS. Subjects viewed images of objects presented at four different orientations (i.e., rotated in depth) on a computer monitor and were asked to press one of two buttons indicating whether the pair of images was the same or different regardless of their orientation. If the second object were perceived to be the same as the first one, neural activity would be decreased. An area cIPS showed the reduction in activation only when two objects were presented at the same orientation. When two objects were presented at different orientations, even though they were the same, area cIPS treated the image from a different viewpoint as a new object. Thus, the orientation of the object with respect to the viewer is critical to control visuomotor actions. However, the ventral stream, and in particular the Lateral Occipital Complex (LOC), showed a reduction in activation for both identical and depth-rotated images of objects. In other words, subjects recognized objects presented even from different viewpoints and LOC, a major part of the ventral stream, plays a role in recognition of objects. The difference in the pattern of activation in LOC and cIPS reflects the difference in the role of the ventral and dorsal stream. The former is for object recognition and the latter is for visuomotor behavior.

The ventral stream is located mainly in the inferotemporal cortex (IT) and adjacent areas including V4 and LOC [15]. fMRI studies on 
humans have shown a positive correlation between the performance of object recognition and cortical activity in the ventral visual region $[27,28]$. The ability to name objects (i.e., recognition performance) showed a similar pattern as the activity in LOC which increases as exposure duration was increased. This correlation was demonstrated by higher activation after training which highly corresponds to enhanced performance on the same stimuli after training. The LOC activation was also stronger in response to trained than novel images consistent with improved performance on recognition of trained images [28]. Those findings of neural activations in the ventral stream located in IT and LOC during recognition of objects have shown that the ventral stream is dissociated from the dorsal stream.

\section{Possible Problems for Visually-guided Action in the Two-system Framework}

Numerous studies have supported the two visual systems theory in which the ventral system is independent from the dorsal system. In sum, the ventral system is involved primarily in object recognition using visual information of an object's features, such as size, orientation and shape, whereas the dorsal system involved primarily in the control and guidance of visuomotor behavior using these same object features. The visual information of object features is transformed into different streams and extracted differently depending on purpose of the performance, recognition or visuomotor behavior. Judgment of 3-D shape is relevant to the ventral system according to the two visual systems theory. However, object shape as well as size, orientation, and location of an object is important to control and guide visuomotor behavior such as grasping movements. For example, when we successfully grasp an object we need to know the location to place the finger at appropriate points on the back of an object. According to two visual systems theorists, the visual information about object shape is analyzed independently for grasping in the dorsal system and for identification (i.e., recognition) in the ventral system [29]. While patient DF with damage to the ventral stream had no difficulty in grasping the object by placing fingers at appropriate points on the object's surface, patient RV with damage to the dorsal stream had trouble [17].

Although it sounds reasonable and persuasive to claim that the visual information about object shape is separately transformed in different systems for different usage, whether the dorsal stream is fully independent from the ventral stream is still in debate. According to the two visual systems theory, different visual streams extract visual information differently and different information is used for recognition of objects and guidance of visuomotor behavior. However, is the ventral stream really separated from the dorsal stream? Some questions arise from the studies supporting the two visual systems theory.

\section{Is Dorsal Stream Automatic and Voluntary Motor Control?}

It has been claimed that the dorsal stream is related to automatic and voluntary motor control [30]. A patient with damage to the dorsal stream, AT, had a difficulty in reaching to a target but her performance improved when her responding was delayed for 5 seconds [31]. In contrast, a patient with damage to the ventral stream, DF had no difficulty in reaching-to-grasp the target immediately, but the errors in performance increased when her responding was delayed [31-33]. The converse pattern of performances in AT and DF have suggested that the dorsal system is dedicated to the immediate guidance of the action using visual information directly, but after a delay visual information long-term coded in the ventral system is required for the action. On the other hand, it has been shown that, in the dorsal stream, the Posterior Parietal Cortex (PPC) is activated during delayed time in order to plan the movement. This finding has confirmed the visuomotor area within the PPC has a specialized role in planning actions such as a saccade, a reach, and a grasping movement $[22-24,34]$.

A question arises when taking these two cases of delayed movements together. However, when the movement is delayed does the dorsal system located mainly in the PPC play a role in planning, but not in guiding movements? How is it that the dorsal stream plans the movement during a delayed time yet does not continue to guide the movement after a delay? It might be possible if we assume that the immediate visual information is used to plan the movement but there is no long-term stored visual information which could be analyzed to guide the movement within the dorsal stream. However, this is not terribly compelling because even a few seconds delay affects the performance. Additionally, two visual systems theorists have assured us that in a delayed condition we use visual information stored in the ventral system to guide actions. If so, could we tell that the dorsal steam is independent from the ventral stream?

\section{Interaction between Two Systems?}

Recently, two visual systems theorists have allowed that the two systems do interact with each other by transferring the information in some way. They still believe, however, that the ventral system is involved in object recognition (perception) while the dorsal system is involved in visual-guided action even though two systems share the information at some level. Furthermore, they try to divide the action part by separating planning from programming of an action [33,34]. Programming of an action involves direct visual information to motor transformations, in which movement parameters are prespecified based on visual information about the object's size, shape, orientation and egocentric position. Planning of an action, however, involves the initial selection of movement (e.g., the type of grip with which the object is grasped or whether to grasp it with one or with two hands [35] based on previous motor experience or stored knowledge about the object to be grasped [36]. In other words, the dorsal stream has a role in on-line control of movement execution in real time and the ventral stream has a role in a perceptual representation of objects based on preserving information about spatial relationships, relative size and orientation and action planning needs perception [34]. Then, is planning of an action distinguished from programming of an action? Although we plan our action based on previous knowledge and motor experiences stored in ventral stream beforehand, we modify our action most of time. Are those actions two separate executions? Additionally, can we not call perception to use visual information of object's size, shape and orientation for on-line control of movement? In short, is perception really separate from action? For instance, when the observer catches the ball, motion of the ball could be the information both for recognition of the ball and for performance in catching the ball. From the pattern of radial expansion and the direction of motion (trajectory motion), we can head to the ball and adjust our position relative to the ball. Also, we need to open our hand and adjust hand orientation appropriately. For adjustment of the hand, we need to perceive the shape of the ball (e.g., baseball or football) and how big the ball is from motion. Thus, perception of 3-D objects is closely related to action. The visually-guided action, such as locomotion and reachesto-grasp, needs perception of 3-D structure in visual space for sure. Can there be perception without action? Basically, we always move our eyes and mostly our head. When forward or side-to-side head 
movements were yielded, reaching performance of distance perception is more accurate and precise [37]. In a similar vein, when participants actively move around a virtual object using a rolling chair [38] or with large perspective changes $\left(\geq 45^{\circ}\right)[39,40]$ they perceived the shape of 3-D objects correctly. Moreover, hand movement interacts with the perception of 3-D objects [41]. Norman et al. [41] investigated whether participants are able to compare naturally shaped 3-D objects (e.g., bell pepper) using their senses of vision and touch. They asked the subjects to actively touch or haptically explore all around the object for a given time, then the subjects judged which one out of 12 objects matched with the object they touched. It has been found that haptic exploration but not visual exploration also produced activation in the lateral occipital cortex (MO), the inferior temporal gyrus [26] and in the lateral occipital complex (LOC) [42]. We can recognize the 3-D object shape from haptic information just like from visual information. Also, the motion of the hand for haptic exploration of object shape produces object recognition just like the motion of the head or body for visual exploration of object shape does. Thus, perception needs action. Again, if perception and action interact with each other, could there be a clear distinction between the ventral and dorsal system?

\section{DCD and the Two Visual Systems Theory}

Although researchers have tried to find the linkage between brain structure and movement deficits in DCD, it is still not straightforward. Nevertheless, some evidence suggests that parietal region is involved in motor performance such as visuospatial processes during motor skill learning [43-45]. For instance, de Olivia and Wann [43] found that DCD group moved significantly slower and was more variable than the control group when they were asked to perform a steering motor task where the visual information was manipulated during motor execution. They suggested that poor visual spatial integration for online movement is due to impaired parietal region. It may be consistent with two visual systems theory in which the dorsal stream is involved in voluntary on-line movement. As I mentioned, however, the distinction between dorsal and ventral stream is questionable. For example, catching a ball is likely to give children with DCD great problems. To reach for a moving object, visual information about the object and it motion, as well as proprioceptive information about the current state of the body parts involved in the reach must be well functioning and integrated. Moreover, prediction of a ball's trajectory should be known. Lefebvre and Reid [46] asked children to predict whether the ball would hit a target or go to the right or left when watching a video for a person throwing a ball towards a target. Children with DCD predicted more poorly compared to a control group and one possible explanation is that visual perceptual problems account for the poor predictive ability. According to the two visual systems theory, the dorsal stream is involved in programming of the movement while the ventral stream is involved in planning of the movement. Then, is prediction of movement programming or planning of the movement?

\section{Conclusion}

In this article I attempt to address a possible limitation of the neurobiological approach in neuropsychiatry, specifically with regards to structural neuroanatomy. This is consistent with a commentary of Dar etal. [47] in which neurobiological approach should be cohesive with other approaches because humans are influenced by many other factors. Although neurobiological approach gives us to solve a long belief of mind brain dualism in psychiatry [48], it can be problematic when we apply certain aspects, in particular regarding the structural and functional neuroanatomy, of that approach in understanding neuropsychiatric phenomena. DCD, for example, is typically related to problems with motor skills but the movement disability is not a solitary problem, rather closely related to perception. The two visual systems theory is prominent when discussing issues of perception and action, and one supported by numerous studies in neuropsychology, neurophysiology, neurobiology, and so on. The proposition of the two visual systems theory is that visual information is transformed in the different visual streams, the dorsal and ventral stream, thus different information is basically used for object recognition and for visuomotor behavior. Also, the ventral stream plays a major role in planning of an action based on previous knowledge and motor experiences while the dorsal stream plays a major role in online visually-guided action. However, as discussed in the article, I believe that there could not be a clear distinction between the dorsal and ventral stream because the same information should be used both for object recognition and visually-guided action in our 3-D visual environment. Ultimately, perception is not so easily separable from action and vice versa. This raises problems in adopting the neuroanatomical basis of the two visual systems theory in addressing DCD, and highlights the limitations of that basis in addressing neuropsychiatric phenomena in general.

In short, researchers try to find distinct function of brain and it helps to understand human behavior and illness but anatomical distinction in neurobiology has its limitations and we must be careful in adopting that approach in neuropsychiatry. Although neurobiological approach could be a good tool for extending psychiatry to clinical neuroscience but it should be collaborated with other dimensions to understand complex neuropsychiatric disorders.

\section{References}

1. Yudofsky SC (2002) Neuropsychiatry and the Future of Psychiatry and Neurology. Am J Psychiatry 159: 1261-1264.

2. Martin JB (2002) The integration of neurology, psychiatry, and neuroscience in the $21^{\text {st }}$ century. Am J Psychiatry 159: 695-704.

3. Price BH, Adams RD, Coyle JT (2000) Neurology and psychiatry: Closing the great divide. Neurology 54: 8-14.

4. Kendler KS (2005) Toward a Philosophical Structure for Psychiatry. Am J Psychiatry 162: 433-440.

5. Wann JP, Mon-Williams M, Rushton K (1998) Postural control and co-ordination disorders: The swinging room revisited. Human Movement Science 17: 491-514.

6. Williams H, Woollacott M (1997) Characteristics of neuromuscular responses underlying posture control in clumsy children. Motor Development; Research and Reviews 1: 8-23.

7. Smits-Engelsman BCM, Niemeijer AS, Van Galen GP (2001) Fine moto deficiencies in children diagnoses as DCD based on poor grapho-motor ability. Human Movement Science 20:161-182.

8. Mon-Williams M, Wann JP, Pascal E (1999) Visual-proprioceptive mapping in developmental coordination disorder. Dev Med Child Neurol 41: 247-254.

9. Sigmundsson H (1999) Inter-modal matching and bi-manual co-ordination in children with hand-eye co-ordination problems. Nordisk Fysioterapi 3: 55-64.

10. Parush S, Yochman A, Cohen D, Gershon E (1998) Relation of visual perception and visual-motor integration for clumsy children. Perceptual and Motor Skills 86: 291-295.

11. Raynor AJ (2001) Strength, power, and coactivation in children with developmental coordination disorder. Dev Med Child Neurol 43: 676-684.

12. Milner AD, Goodale MA (1995) The visual brain in action, Oxford: Oxford University Press.

13. Ungerleider LG, Mishkin M (1982) Two cortical visual systems. In Ingle DJ, Goodale MA, Mansfield RJW (Eds.). Analysis of Visual Behavior 549-586). MIT Press Cambridge MA.

14. Goodale MA, Milner AD (1992) Separate visual pathways for perception and action. Trends in Neurosciences 15: 20-25. 
Citation: Lee Y (2015) Limitations of Neurobiological Approach in Neuropsychiatry: DCD and the Two Visual Systems Theory. J Psychiatry 18: 289 doi: $10.4172 / 2378-5756.1000289$

Page 5 of 5

15. Norman J (2002) Two visual systems and two theories of perception: An attempt to reconcile the constructivist and ecological approaches. Behav Brain Sci 25: 73-144.

16. Prenin M-T, Vighetto A (1988) Optic ataxia: a specific disruption in visuomotor mechanisms. I. Different aspects of the deficit in reaching for objects. Brain 111: 643-674.

17. Goodale MA, Meenan JP, Bülthoff HH, Nicolle DA, Murphy KJ, et al. (1994) Separate neural pathways for the visual analysis of object shape in perception and prehension. Current Biology 4: 604-610.

18. Jakobson LS, Archibald YM, Carey DP, Goodale MA (1991) A kinematic analysis of reaching and grasping movements in a patient recovering from optic ataxia. Neuropsychologia 29: 803-809.

19. Jeannerod M, Decety J, Michel F (1994) Impairment of grasping movements following a bilateral posterior parietal lesion. Neuropsychologia 32: 369-380.

20. Goodale MA, Milner AD, Jakobson LS, Carey DP (1991)A neurological dissociation between perceiving objects and grasping them. Nature 349: 154-156.

21. Cohen YE, Andersen RA (2002) A common reference frame for movement plans in the posterior parietal cortex. Nature Reviews Neuroscience 3: 553-562.

22. Snyder LH, Batista AP, Andersen RA (1997) Coding of intention in the posterior parietal cortex. Nature 386: 167-170.

23. Murata A, Gallese V, Kaseda M, Sakata H (1996) Parietal neurons related to memory-guided hand manipulation. J Neurophysiol 75: 2180-2186.

24. Connolly JD, Andersen RA, Goodale MA (2003) FMRI evidence for a 'parietal reach region'in the human brain. Exp Brain Res 153: 140-145.

25. Culham JC, Dnackert SLD, DeSouza JFX, Gati JS, Menon RS, et al. (2003) Visually guided grasping produces fMRI activation in dorsal but not ventral stream brain areas. Experimental Brain Research 153: 180-189.

26. James TW, Humphrey GK, Gati JS, Servos P, Menon RS, et al. (2002) Haptic study of three-dimensional objects activates extrastriate visual areas. Neuropsychologia 40: 1706-1714.

27. Bar M, Tootell RBH, Schacter DL, Greve DN, Fischl B, et al. (2001) Cortical mechanisms specific to explicit visual object recognition. Neuron 29: 529-535.

28. Grill-Spector K, Kushnir T, Hendler T, Malach R (2000) The dynamics of objectselective activation correlate with recognition performance in humans. Nature Neuroscience 3: 837-843.

29. Goodale MA, Jakobson LS, Servos $P$ (1996) The visual pathways mediating perception and prehension. In Wing AM, Haggard P, Flanagan JR (Eds.), Hand and Brain: The neurophysiology and psychology of hand movements (pp. 1531). San Diego CA: Academic Press Inc.

30. Pisella L, Gréa H, Tilikete C, Vighetto A, Desmurget M, Rode G, Boisson D, Rossetti $Y$ (2000) An 'automatic pilot'for the hand in human posterior parietal cortex: toward reinterpreting optic ataxia. Nature Neuroscience 3: 729-736.

31. Milner AD, Dijkerman HC, Carey DP (1999) Visuospatial processing in a pure case of visual-form agnosia. In Burgess $\mathrm{N}$, Jeffery KJ, O'keefe J (Eds.), The hippocampal and parietal foundations of spatial cognition (pp. 443-466). Oxford University Press.
32. Goodale MA, Jakobson LS, Keillor JM (1994) Differences in the visual control of pantomimed and natural grasping movement. Neuropsychologia 32: 1159-1178.

33. Goodale MA, Milner AD (2004) Plans for action. Behav Brain Sci 27: 37-40.

34. Milner AD, Goodale MA(2008) Two visual systems re-viewed. Neuropsychologia 46: 774-785.

35. van Doorn H, van der Kamp J, Savelsbergh GJP (2007) Grasping the Muller-Lyer illusion: The contributions of vision for perception in action. Neuropsychologia 45: 1939-1947

36. Carey DP, Harvey M, Milner AD (1996) Visuomotor sensitivity for shape and orientation in a patient with visual form agnosia. Neuropsychologia 34: 329-337.

37. Wickelgren EA, McConnell DS, Bingham GP (2000) Reaching measures of monocular distance perception: Forward versus side-to-side head movements and haptic feedback. Perception \& Psychophysics 62: 1051-1059.

38. Bingham GP, Lind M (2008) Large perspective transformations are necessary and sufficient for accurate perception of metric shape. Perception \& Psychophysics 70: 524-540.

39. Lee $Y$, Bingham GP (2010) Large perspective changes $\left(\geq 45^{\circ}\right)$ yield perception of metric shape that allows accurate feedforward reaches-to-grasp, and it persists after the optic flow has stopped! Exp Brain Res 204: 559-573.

40. Lind M, Lee Y, Mazanowski J, Kountouriotis GK, Bingham GP (2014) Affine operations plus symmetry yield perception of metric shape with large perspective changes $\left(\geq 45^{\circ}\right)$ : Data and model. J Exp Psychology: Hum Percept Perform 40: 83-93.

41. Norman JF, Norman HF, Clayton AM, Lianekhammy J, Zielke G (2004) The visual and haptic perception of natural object shape. Perception \& Psychophysics 66: 342-351.

42. Amedi A, Malach R, Hendler T, Peled S, Zohary E (2001) Visuo-haptic objectrelated activation in the ventral visual pathway. Nature Neuroscience 4: 324-330.

43. de Olivia RF, Wann JP (2010) Integration of dynamic information for visuomotor control in young adults with developmental coordination disorder. Experimental Brain Research 205: 387-394

44. Kashiwagi M, Iwaki S, Narumi Y, Tamai H, Suzuki S (2009) Parietal dysfunction in developmental coordination disorder: A functional MRI study. Neuroreport 20: $1319-1324$

45. Zwicker JG, Missiuna C, Harris SR, Boyd LA (2011) Brain activation associated with motor skill practice in children with developmental coordination disorder: An fMRI study. Int J Dev Neurosci 29: 145-152.

46. Lefebvre C, Reid G (1998) Prediction in ball catching by children with and without developmental coordination disorder. Adapted Physical Activity Quarterly 15: 299-315.

47. Dar MA, Wani RA, Rather YH (2015) An anti-thought about neurobiological approach in psychiatry. J Pscyhiatry 18:183.

48. Raese $J$ (2015) The pernicious effect of mind/body dualism in psychiatry. $J$ Psychiatry 18: 219 